\title{
Cognitive Sensitivity and Dream Recall in Children
}

\author{
Sudhakar Venukapalli \\ Department of Education, The English and Foreign Languages University, Hyderabad, India
}

\section{Email address:}

sudhakarvenu.efluniversity@gmail.com

\section{To cite this article:}

Sudhakar Venukapalli. Cognitive Sensitivity and Dream Recall in Children. American Journal of Applied Psychology.

Vol. 10, No. 2, 2021, pp. 34-39. doi: 10.11648/j.ajap.20211002.11

Received: March 28, 2021; Accepted: April 14, 2021; Published: April 23, 2021

\begin{abstract}
Does an individual's inner cognitive sensitivity influence his /her recalling of dreams? Do girls recall more number of dreams than boys? This paper is an attempt to address these two questions through an empirical study conducted with 40 school children. Recent studies in dream research are enabling the scientific community to go deep into the complex cognitive processes, including the functioning of the whole personality. The dream recall questionnaire and the Cognitive Process Survey (CPS) designed and developed by Raymond F. Martinetti were circulated among the children and their responses were processed and analyzed. The data about dream recall were also crosschecked with dream diaries that were maintained by the children during the study period. It is found that boys and girls differ significantly with respect to the recalling of dreams and the most interesting finding of this study is that girl's recall more number of dreams compared to boys. Further, the Cognitive Process Survey (CPS) assessed children's inner cognitive sensitivity like, degree of imaginal life, orientation toward imaginal life and defensiveness. This study also discovered a strong and positive correlation between dream recall and the inner imaginal sensitivity of children. The findings of this study are consistent with the studies conducted by cognitive psychologists and clinical psychologists in the field of dream research.
\end{abstract}

Keywords: Dream Recall, Dream Analysis, Cognitive Sensitivity, Imaginal Life, and Cognition

\section{Introduction}

One of the fascinating questions in contemporary cognitive psychology is what factors contribute to dreaming. Researchers with different disciplinary backgrounds are making serious attempts to address this question. Cognitive Neuropsychologists and Bio-psychologists have been focusing their attention on the variables such as type of sleep (REM sleep and non-REM sleep), phasic vs. tonic, waking thresholds etc. On the other hand, cognitive psychologists are examining the influence of various psychological variables like anxiety, defense mechanisms, social interests, cognitive styles, memory, intelligence, etc.

Dreaming is an integral part of human existence. It is purely a subjective and personal experience and a state of consciousness regulated by internal and external factors. Dreams consist of images, symbols, monologues, dialogues, conversations, people, the world of plants and animals and imaginary persons, objects, relations, events, and experiences. Dreams are like playhouses, dramaturgy, and theaters. Why do people dream and what factors influence dream productivity is still a largely unanswered question.

Individuals differ greatly in dreaming and recalling their dreams. Why do some people remember their dreams, while others don't? Why do some people remember more of their dreams? Why do individuals differ in remembering dreams? What individual differences exist in remembering dreams? Do cognitive factors regulate the memory and recall of dreams? Why individuals differ in their ability to remember dreams? These are a few important questions in the contemporary dream research $[1,2]$

While exploring answers to the above questions the researchers in the field of cognitive psychology of dream research got fascinated with the influence of inner cognitive sensitivity on dreaming and dream recall $[3,4]$. Many studies observed a positive relationship between the ability of dream recall and the sensitivity of an individual towards inner cognitive states. The research studies conducted by Schonabar [5] and Hiscock \&Cohen [6] supported the hypothesis that individuals dream recall is associated with inner states of cognitive sensitivity. 


\section{Methodology}

Considering various research studies in cognitive psychology and dream research the following research questions and hypotheses are formulated.

\subsection{Research Questions and Hypotheses}

1) Does sex play any role in dream recall and cognitive sensitivity?

2) Does an individual's sensitivity to inner states influence the ability of dream recall?

As discussed above many studies have reported the correlation between different cognitive aspects of imaginal life and dreams recalled. Previous researchers hypothesized that higher the sensitivity to inner states higher will be the dream recall and emotional dream content. The probability of recall is a function of cognitive and emotional factors. Dream research also discovered that more the suppression of anger and sexual ideation more would be the recalling of dreams. Considering the background research and intensive literature survey, the following hypotheses are formulated in this investigation.

i. Girls recall more number of dreams than boys.

ii. Boys and girls differ significantly with respect to recalling of their dreams

iii. There is a significant difference between high, average and low dream recallers with respect to the variability of dreams recalled

iv. Girls and boys differ significantly with respect to the degree of imaginal life.

v. Girls and boys differ significantly with respect to the degree of orientation towards imaginal life.

vi. Girls and boys do not differ significantly with respect to the degree of suppression/ defensiveness.

vii. There exists a strong positive correlation between dream recall and children's degree of imaginal life.

viii. Dream recall is positively correlated with the degree of emotional response to imaginal experiences.

ix. There exists a strong positive correlation between children's degree of suppression/defensiveness and their dream recall.

\subsection{Participants}

A group of forty (40) school children studying class VII in Hyderabad city schools were identified through convenience and snowball sampling technique. The average age of this group of children was 12 years. All the parents of the children were literates having a minimum 15 years of schooling background. Very few parents were having 20 years of formal education. The children of this sample were from a middle class urban background. Children's and parents consent was taken to participate in the study. Parents of children supported the researcher and the study environment. All children of the study were healthy and normal. Children and their parents were briefed about the purpose of the study and method and procedures of data collection.

\subsection{Instruments}

The approach of this study is basically quantitative and non-experimental descriptive research. The researcher administered the following three instruments for collecting the data.

i. Dream Recall Questionnaire (DRQ)

ii. Cognitive Processes Survey (CPS)

iii. Dream Diary (DD)

\subsection{Dream Recall Questionnaire (DRQ)}

The dream recall questionnaire was administered on the sample of school children. This tool comprises a five-point rating scale. The reliability of this scale for an average interval of 30 days is $r=0.78$.

How often have you recalled your dreams recently (in the past several months)?

A. more than six dreams a week

B. five to six dreams a week

C. two or four dreams a week

D. about once a week

E. never

The dream recall frequency is calculated on the basis of 5 point scale. The scores assigned to the above categories are: $0=$ never, 1 = about once a week, 2 = two or four dreams a week, $3=$ five or six dreams a week, and $4=$ more than six dreams a week

\subsection{Cognitive Processes Survey (CPS)}

This tool was developed by Raymond F. Martinetti [7] to measure the cognitive components of imaginal life. The CPS may be useful for exploring the continuity of cognitive imaginal processes between waking and sleep. The 39- item Likert type survey, where the participants were asked to respond to each item by marking (SA) Strongly Agree, (A) Agree, (U) Undecided, (D) Disagree, or (SD) Strongly Disagree, in the spaces provided. This survey instrument measures three important components of imaginal activity of children. The three components are:

i. The Degree of Imaginal Life. It is about the intensity and extensity of imaginal activity. The basic objective here is to measure (a) "imaginal life or the extent to which the person engages in imagery, fantasy and other forms of introspection" [7]. For this purpose thirteen (13) statements are framed. It is assumed that high scorers of the degree of imaginal life "tend to have high dream recall, complex dreams, and a high capacity to experience multisensory arousal" [8]

ii. Orientation Toward Imaginal Life. "A person's acceptance or denial of inner experience"[7]. What orientation people take toward inner experience. Do they deny or accept their inner experience? Thirteen (13) statements are verbalized for measuring an individual's emotional response to imaginal processes.

iii. Degree of Suppression/Defensiveness. This aspect of 
cognitive process survey represents "defensiveness or the extent to which the person denies conscious awareness of distressing or socially unacceptable thoughts" [7]. To measure "the tendency to suppress feelings, especially anger and sexual ideation"[8] thirteen (13) statements are designed.

As mentioned above the CPS is scored on a 5-point Likert scale along an equal interval continuum from "strongly agree " to "strongly disagree" with scores for each subscale being a simple sum of the items on the subscale and scores for the total scale being a sum of all 39 items. Reliability coefficients for the three subscales are reported as 0.78 for the degree of imaginal life, 0.75 for the orientation toward imaginal life., and 0.72 for degree of suppression or defensiveness.

\subsection{Procedures of Data Collection}

After developing rapport with the parents and children the researcher individually briefed them about the process of the study and their sensitive role in data collection. Dream diaries were circulated among the participants with clear instruction. Children were asked to recall and record their dreams just after the morning awakenings. Parents were requested to support in reminding children about their daily recordings without interfering with the children's dream experiences. Dreams were collected from all the children of the sample for 45 days. This home based method of data collection worked out very well in obtaining the data. For the purpose of reliability of the data, the frequency of dreams recalled was collected from dream diaries and it was crosschecked with the Dream Recall Questionnaire.

\section{Results and Discussion}

The data collected through the Dream Recall Questionnaire (DRQ) was categorized into three groups. Participants who recalled 5 and above dreams per week were placed in the High Dream Recallers category, participants recalled 4 to 2 dreams per week were placed in the Average Dream Recallers category and, the participants recalled one and less than one dreams per week were placed in the Low Dream Recallers category. From the collected data, it is observed that in high dream recall category there are 11 girls and 8 boys; 7 boys and 7 girls in average dream recall category and 2 girls and 5 boys are found in low dream recall category. This data is presented in a tabular form below.

Table 1. Distribution of students with respect the number of dreams recalled per week.

\begin{tabular}{llll}
\hline Dream Recall & Range/Number of Dreams Recalled per week & N & Mean \\
\hline High & 5 and above & 19 (11Girls+8Boys) & 6.42 \\
Average & $4-2$ & 14 (7Girls+7 Boys) & 1.86 \\
Low & $1-0$ & 07 (2Girls+5Boys) & 0.73 \\
\hline
\end{tabular}

The above-presented distribution of dream recall with respect to the categories high, average, and low dream recall clearly show that more number of girls is from high dream recallers compared to the boys. This supports the hypothesis (i).
The question whether high, medium, and low dream recallers differ significantly was addressed by administering the one-way ANOVA statistical test and the results are presented below.

Table 2. One-way ANOVA.

\begin{tabular}{|c|c|c|c|c|c|}
\hline Source of Variation & Sum of Squares & d.f & Variance & $\mathbf{F}$ & $\bar{p}$ \\
\hline Between Groups & 189.3834 & 2 & 94.7789 & & \\
\hline Within Groups & 70.0669 & 37 & 1.9032 & 49.80052 & 0.0000 \\
\hline TOTAL & 259.4503 & 39 & & & \\
\hline
\end{tabular}

The F-ratio value is 49.80052 . The $\mathrm{p}$-value is $<.00001$. The result is significant at $\mathrm{p}<.05$.

From the above analysis it is inferred that there is a significant difference between high, average and low dream recallers. This supports the acceptance of the Hypothesis (iii).

Gender-wise analysis of the three categories of dream recall is presented along with the mean differences and t-test values.

Table 3. Gender Vs. High, Average and Low Dream Recall.

\begin{tabular}{|c|c|c|c|c|c|c|c|}
\hline CPS & Gender & Mean & SD & SE of difference & df & t-value & p-value(2 tailed) \\
\hline \multirow[b]{2}{*}{ High Dream Recall } & Girls (11) & 7.27 & 2.20 & \multirow{2}{*}{0.814} & \multirow{2}{*}{17} & \multirow{2}{*}{2.34} & $0.0151 *$ Sig. \\
\hline & Boys (8) & 5.38 & 0.74 & & & & $\mathrm{p}>0.05$ \\
\hline \multirow{2}{*}{$\begin{array}{l}\text { Average Dream } \\
\text { Recallers }\end{array}$} & Girls & 3.14 & 0.69 & \multirow{2}{*}{0.404} & \multirow{2}{*}{12} & \multirow{2}{*}{0.353} & 0.3645 Not Sig. \\
\hline & Boys & 3.0 & 0.82 & & & & $\mathrm{p}<0.05$ \\
\hline \multirow{2}{*}{ Low Dream Recallers } & Girls & 1.0 & 0.00 & \multirow{2}{*}{0.427} & \multirow{2}{*}{5} & \multirow{2}{*}{0.63} & 0.2736 Not Sig. \\
\hline & Boys & 0.8 & 0.45 & & & & $\mathrm{p}<0.05$ \\
\hline
\end{tabular}

From the above table, it is observed that there are significant differences between girls and boys belonging to the high dream recall category. However no differences between boys and girls are found in the other two categories i.e., Average and Low dream recall. The over all dream recall data was analyzed with respect to the sex background of the students and the results are 
presented below.

Table 4. Gender Vs. Overall Dream Recall.

\begin{tabular}{|c|c|c|c|c|c|c|c|}
\hline Measure & Gender & Mean & SD & SE Difference between Means & d.f & t-value & p-value (two tailed) \\
\hline \multirow{2}{*}{ Dream Recall } & Girls & 5.10 & 2.93 & \multirow{2}{*}{0.7907} & \multirow{2}{*}{38} & \multirow{2}{*}{2.25258} & \multirow{2}{*}{$.030142 *$ Sig. } \\
\hline & Boys & 3.40 & 1.98 & & & & \\
\hline
\end{tabular}

From the above table it is clearly evident that the mean value of dream recall is higher compared to their counterparts. From this analysis, it is inferred that girls scored higher than boys on dream recall. The t-value is 2.25258. The $\mathrm{p}$-value is .030142 . The result is significant at $\mathrm{p}$ $<.05$. It is inferred from this table that girls and boys differ significantly with respect to the recalling of dreams. The Welch test is a modified version of t-test used when the variances of the groups are not same. Welch's t-test results are also similar to this finding. Welch's t-test: $\mathrm{p}=0.03087$ $(\mathrm{t}=2.25258 ; \mathrm{df}=33.90101)$. Based on Welch's t-test, as $\mathrm{p}<0.05$, the null hypothesis of equal means is rejected. This supports the hypothesis (ii).

\section{Effect size (Cohen's d for Welch test)}

Since the variance of dream recall in boys and girls are not equal (unequal standard deviations), the Welch's t-test, which is more flexible compared to the Student's t-test was also adopted to calculate the effect size. The effect size can be computed by dividing the mean difference between the groups by the "averaged" standard deviation. The observed standardized effect size is large (0.68). That indicates that the magnitude of the difference between girls and boys is large. The Welch's test is based on the assumption that the variance is not the same in the two groups, which results in the fractional degrees of freedom.

Students responses on the statements of Cognitive Process Survey were also collected and the intensity of imaginal life, orientation toward imaginal life and defensiveness were measured and analyzed. The possible range of scores for each subscale was 13 to 65 . Means and standard deviation values were calculated category and theme-wise and the data is presented below.

Table 5. Three dimensions of the Cognitive Process Survey.

\begin{tabular}{llll}
\hline Measure & CPS & Mean & SD \\
\hline \multirow{2}{*}{ Cognitive Process } & Degree of Imaginal Life & 38.18 & 5.93 \\
Survey & Degree of Emotional Response to Imaginal Experiences & 33.61 & 5.86 \\
& Degree of Suppression and Defensiveness & 27.17 & 2.71 \\
\hline
\end{tabular}

Table 6. One-way ANOVA.

\begin{tabular}{lllll}
\hline Source of Variation & Sum of Squares & d.f & Variance & F \\
\hline Between Groups & 2447.7147 & 2 & 1223.8573 & \\
Within Groups & 2997.0954 & 117 & 25.6162 & 0.0000 \\
TOTAL & 5444.8101 & 119 & & 47.78 \\
\hline
\end{tabular}

Tukey HSD Post-hoc Test.

Group 1 vs Group 2: Diff $=-4.5700,95 \% \mathrm{CI}=-7.2565$ to -1.8835 , $\mathrm{p}=0.0003$

Group 1 vs Group 3: Diff $=-11.0100,95 \% \mathrm{CI}=-13.6965$ to $-8.3235, \mathrm{p}=0.0000$

Group 2 vs Group 3: Diff $=-6.4400,95 \% \mathrm{CI}=-9.1265$ to -3.7535 , $\mathrm{p}=0.0000$

It is observed from the above table that the $\mathrm{F}$ value is 47.78 at $2 \& 117$ degrees of freedom and the $p$ value is less than 0.05 . These results clearly indicate that the three aspects of Cognitive Sensitivity (i. degree of imaginal life, ii. degree of emotional response to imaginal experiences and, iii. degree of suppression and defensiveness.) are distinct categories. This result is in consistent with research studies conducted by previous cognitive psychologists like [7].

As discussed above the cognitive process survey was administered on school children to know whether boys and girls differ with respect to the three components of the cognitive sensitivity. The data analyzed is presented below.

Table 7. Gender Vs. Cognitive Process Survey.

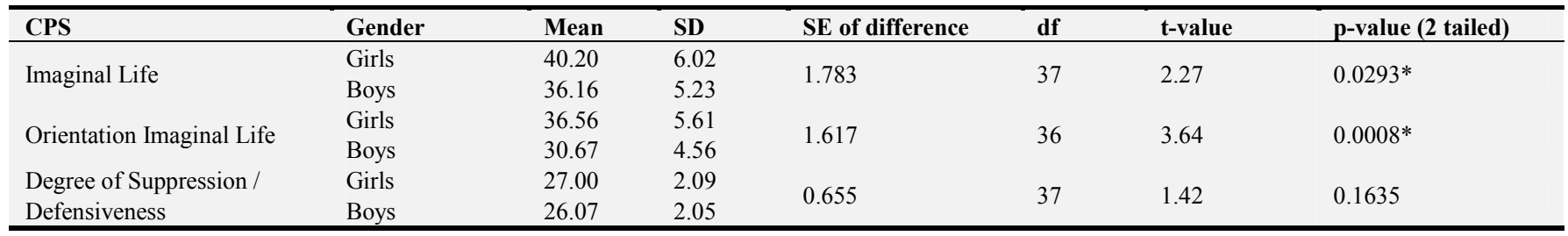

In the case of Imaginal Life the Welch's t-test statistical analysis shows a significant difference between girls and boys. The $\mathrm{p}$-value equals $0.0293716,(\mathrm{p}(\mathrm{x} \leq \mathrm{T})=0.985314)$. This means that the chance of type 1 error is small: 0.02937
$(2.94 \%)$. The observed standardized effect size is large (0.72). That indicates that the magnitude of the difference between the girls and boys is large. Since $p$-value $<\alpha$, the null hypothesis is rejected. The average of girls population is 
considered to be not equal to the average. of the boys population. In other words, the difference between the average of the girl's and boy's populations is big enough to be statistically significant. Hence, girls and boys differ significantly with respect to the degree of imaginal life.

With reference the Orientation towards Imaginal Life the Welch's t-test statistical analysis indicates a significant difference between girls and boys. The p-value equals 0.000830661, $(\mathrm{p}(\mathrm{x} \leq \mathrm{T})=0.999585)$. This means that the chance of type 1 error is small: $0.0008307(0.083 \%)$.

The observed standardized effect size is large (1.15). That indicates that the magnitude of the difference between the average and average is large. Since p-value $<\alpha$, the null hypothesis is rejected. The average of girl's population is considered to be not equal to the average of the boy's population. Hence, the difference between the average of the girls and boys populations is big enough to be statistically significant. Hence, girls and boys differ significantly with respect to the degree of attitude towards orientation of imaginal life.

Results in the case of the Degree of Suppression / Defensiveness showed different results. From the Welch's ttest analysis it is inferred that there is no significant difference between boys and girls. The test statistic $\mathrm{T}$ equals 1.420667 , is in the $95 \%$ critical value accepted range: [2.0244: 2.0244]. The p-value equals $0.163571,(\mathrm{p}(\mathrm{x} \leq \mathrm{T})=$
0.918215). This means that if we would reject the null hypothesis, the chance of type I error would be too high: $0.1636(16.36 \%)$. The larger the p-value the more it supports $\mathrm{H} 0$. The observed standardized effect size is medium (0.45). That indicates that the magnitude of the difference between the average and average is medium. Since p-value $>\alpha$, the null hypothesis is accepted. The average of girl's population is considered to be equal to the average of the boy's population. In other words, the difference between the average of the girls and boys populations is not big enough to be statistically significant. Hence, girls and boys do not differ significantly with respect to the degree of suppression /defensiveness.

The above results clearly showed that subjects with the highest degree of imaginal life and orientation towards imaginal life reported most dreams recalled. It is also found that the degree of suppression showed no difference on dream recall across the gender. The research findings of T. L. Cory, W. Ormistoni, E. Simmel, \& M. Dainoff [9], Spanos, H J. Stam, H. L. Radtke and M. E. Nightingle [10] and Raymond F. Martinnetti [7, 8] corroborate the findings of this study.

Pearson product moment correlation coefficient (r) values are calculated to measure the strength of relationship between cognitive sensitivity and dream recall in girls and boys. All the six values are presented in the following table.

Table 8. Pearson Correlation between Dream Recall and Cognitive Sensitivity in girls and boys.

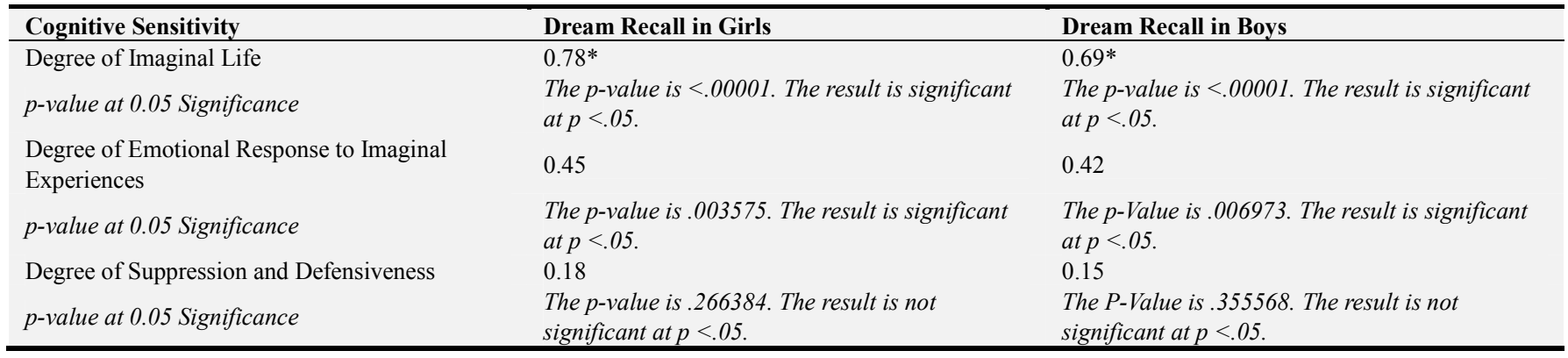

The above table indicates that there exists both in the case of girls and boys a strong positive correlation between dream recall and children's degree of imaginal life. Dream recall is also positively correlated with the degree of emotional response to imaginal experiences. Very insignificant correlation is observed between children's degree of suppression /defensiveness and their dream recall. The results of this study support the findings of previous studies that girls recall dreams more often than boys. Foulkes, Shepherd, and Scott [11] and Foulkes, Petrik, and Scott [12] revealed that girls tend to recall their dreams more often than boys. The results of this study are also in consistent the findings of the studies conducted by Domino [13]; Schredl, Nurnberg \&Weiler [4]; Schredl (2000) and, Schredl and Piel [14]

\section{Conclusions}

From this empirical study, it is inferred that the inner cognitive sensitivity of an individual and dreaming are correlated and most importantly individual's dream recalling is a function of his/her inner imagination and attitude or emotional response towards imaginal life. It is also observed that children who are rich in imaginative life are able to recall more number of dreams compared to others. The results of this study corroborate the findings of Raymond F. Martinetti [7, 15, \& 16] and many other studies conducted by the cognitive psychologists. Dreaming is a complex sociobiological process where many cognitive and noncognitive factors influence, shape, and manufacture them. One needs to understand the limits of this investigation by recognizing such complexities and dynamics of dreaming.

\section{References}

[1] Blagrove, Mark. "Problems with the Cognitive Psychological Modeling of Dreaming", The Journal of Mind and Behaviour, Vol. 17, No. 2, pp. 99-134, 1996. 
[2] Blagrove, Mark. "Gender Differences in the Dream Content of Children and Adolescents", The American Journal of Psychology, Volume: 132, Pages: 315-324, 2019.

[3] Van De Castle, R,. L. The Psychology of Dreaming. New Jersey: General Learning Press, 1971.

[4] Schredl, M., Nurnberg, C., \& Weiler, S. "Dream recall, attitude toward dreams, and personality", Personality and Individual Differences, 20, 613-618, 1996.

[5] Schonbar R., A. "Some manifest characteristics of recallers and non-recallers of dreams", Journal of Consulting Psychology, 23, 414-418, 1959.

[6] Hiscock, M., \& Cohen, D. B. "Visual imagery and dream recall, Journal of Research in Personality", 7, 179-188, 1973.

[7] Martinetti, R, F. "Cognitive antecedents of dream recall", Perceptual and Motor Skills, 60, 395-401, 1985.

[8] Martinetti, R, F., "Sex differences in dream recall and Components f Imaginal Life", Perceptual and Motor Skills, 69, 643-649. 1989.

[9] Cory, T. L., Ormistoni, W., Simmel, E., \& Dainoff, M. "Predicting the frequency of dream recall", Journal of Abnormal Psychology, 84, 261-266, 1975.

[10] Spanos, N. P., Stam, H. J., Radtke H. L and Nightingle, M. E. "Absorption in Imaginings, Sex-Role Orientation, and the Recall of Dreams by Males and Females", Journal of Personality Assessment, 44, 277-282, 1980.

[11] Foulkes, D., Shepherd, J., \& Scott, E. “Analysis of children's dreams at ages 5-6 and 11-12", Sleep Research, 3, 117, 1974.
[12] Foulkes, D., Petrik, J., \& Scott, E. A. “Analysis of children's dreams at ages 7-8 and 13-14". Sleep Research, 7, 175, 1978.

[13] Domino, G. "Attitudes toward dreams, sex differences, and creativity", Journal of Creative Behavior, 16, 112-122, 1982.

[14] Schredl, M., Edgar Piel. "Gender differences in dream recall: data from four representative German samples", Personality and Individual Differences 35, 1185-1189, 2003.

[15] Martinetti, R, F. "Dream recall, Imaginal processes and shortterm memory, a pilot study". Perceptual and Motor Skills, 57, $718,1983$.

[16] Cohen D. B. "Toward a theory of dream recall", Psychological Bulletin, 81, 138-154, 1974.

\section{Biography}

Sudhakar Venukapalli is currently working as a Professor in the Department of Education and DEAN of the School of English Language Education in the English and Foreign Languages Central University, Hyderabad, India. Dr. Sudhakar Venukapalli has a diverse educational background spanning physics, philosophy, psychology, psychotherapy, and education. His contributions are primarily in the area of Philosophy of Science Cognitive Psychology, Psycholinguistics, and Education and Policy Studies. Dr. Sudhakar Venukapalli is also a consultant to the World Bank, DFID, UNICEF, and he is a visiting faculty to a number of universities and civil society organizations. 